FORMATION Formation emploi

Revue française de sciences sociales

94 | avril-juin 2006

Immigration et marché du travail

\title{
Les immigrés accèdent moins à la formation professionnelle continue
}

Immigrants have less access to continuing vocational training

Migranten: Seltener Zugang zu Weiterbildungsmaßnahmen

Didier Gélot et Claude Minni

\section{CpenEdition}

Journals

Édition électronique

URL : http://journals.openedition.org/formationemploi/2423

DOI : 10.4000/formationemploi.2423

ISSN : 2107-0946

Éditeur

La Documentation française

Édition imprimée

Date de publication : 1 avril 2006

Pagination : 93-109

ISSN : 0759-6340

Référence électronique

Didier Gélot et Claude Minni, « Les immigrés accèdent moins à la formation professionnelle continue », Formation emploi [En ligne], 94 | avril-juin 2006, mis en ligne le 04 décembre 2009, consulté le 30 octobre 2020. URL : http://journals.openedition.org/formationemploi/2423 ; DOI : https://doi.org/ 10.4000/formationemploi.2423

(c) Tous droits réservés 


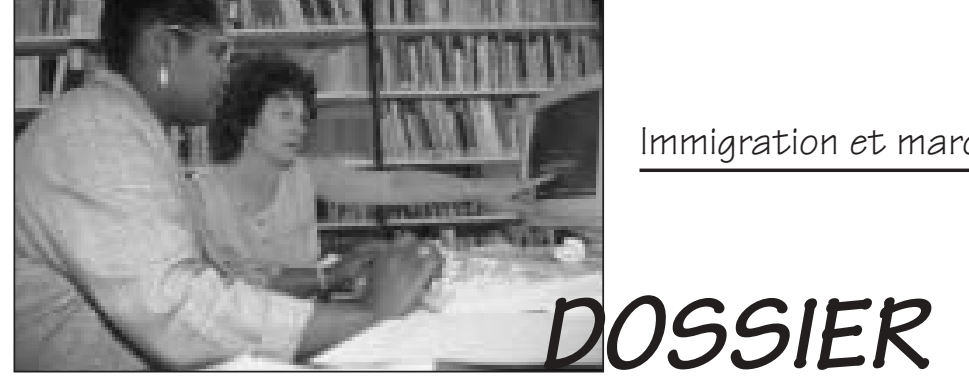

\section{Les immigrés accèdent moins à la formation professionnelle continue}

par Didier Gélot et Claude Minni*

Les immigrés accèdent deux fois moins à la formation professionnelle continue que les Français d'origine. En revanche, les bénéficiaires qui sont dans ce cas suivent des actions plutôt plus longues et plus qualifiantes que la moyenne des stagiaires. Les personnes nées de parents immigrés, quant à elles, accèdent dans des proportions proches de celles des non-immigrés aux dispositifs de formation. Seules celles dont les deux parents sont originaires du Maghreb apparaissent défavorisées.

De nombreux travaux ont abordé les discriminations envers les populations immigrées ou issues de l'immigration $^{1}$, que ce soit en termes de scolarisation ou de cheminement dans l'emploi. En revanche, on ne trouve pas en France d'études portant sur l'accès des immigrés à la formation professionnelle continue ou aux autres formations post-scolaires (formations sous statut de chômeur ou formations suivies à titre individuel). Il est vrai que la question des « personnes d'origine étrangère » est relativement inédite dans la statistique publique française. Il y a une quinzaine d'années

\footnotetext{
${ }^{1}$ Dans son rapport de 1991, le Haut Conseil à l'Intégration, s'appuyant sur les travaux de Michèle Tribalat (1989), a proposé d'appeler « immigrés » les personnes nées à l'étranger, entrées sur le territoire avec une nationalité étrangère et résidant en France depuis un an au moins. Conséquence de cette définition, les enfants d'immigrés, s'ils sont nés en France, ne sont pas eux-mêmes immigrés. Le terme d'immigrés est lui-même sujet à caution, puisque d'un point de vue purement juridique seule prévaut la notion d'étrangers. Il s'agit essentiellement d'une convention à des fins d'études (Héran, 2002).
}

* Didier Gélot, lors de la rédaction de cet article, était adjoint à la sous-direction du suivi et de l'évaluation des politiques d'emploi et de formation professionnelle de la DARES (Direction de l'animation de la recherche, des études et des statistiques au ministère chargé de l'Emploi et de la Formation professionnelle). II a notamment publié : "Le rôle de l'encadrement intermédiaire dans la formation en entreprise : entre rhétorique gestionnaire et réalité sociologique », Travail et Emploi (2006); Pour l'éducation permanente: propositions pour la formation des salariés et des chômeurs, Éditions Syllepse (2005).

Claude Minni est chargé d'études au département Emploi de la DARES. II s'intéresse plus particulièrement à l'insertion professionnelle des jeunes, ainsi qu'à la place des seniors sur le marché du travail. Sur ces thèmes, il a notamment publié dans Économie et Statistique : "L'emploi des jeunes au coeur des dynamiques du marché du travail » (2005) et « Les entreprises face au vieillissement de leurs effectifs » (2003). 
encore, les études qui cherchaient à mesurer certains phénomènes socio-économiques propres à ces populations s'appuyaient plutôt sur la nationalité et négligeaient les étrangers devenus français, ainsi que ceux nés en France dont les parents sont d'origine étrangère (cf. encadré 1).

\section{Encadré 1 \\ Immigrés et personnes issues de l'immigration}

On distingue ici les immigrés et leurs descendants directs. Les premiers sont les personnes nées étrangères à l'étranger. Certaines sont de nationalité étrangère à la date de l'enquête, d'autres sont devenues françaises par naturalisation. Les seconds sont des personnes non immigrées, dont un au moins des deux parents est né étranger à l'étranger. L'enquête FQP (Formation et qualification professionnelle) 2003 comptabilise 36,4 millions de personnes âgées de 17 à 65 ans. Parmi celles-ci, 3,4 millions sont des immigrées et 2,6 millions sont issues de l'immigration. 1,5 millions d'immigrés sont originaires d'un pays d'Europe, 1 million d'un pays du Maghreb (Algérie, Maroc ou Tunisie), près de 400000 d'un autre pays d'Afrique, un peu plus de 100000 d'un pays d'Amérique, et à peu près le même nombre $d^{\prime}$ Asie du Sud-Est (Cambodge, Laos, Vietnam).

Les personnes nées de parents immigrés se répartissent à parts égales entre familles mixtes et familles dont les deux parents sont immigrés. C'est le cas de 400000 personnes dont les deux parents sont maghrébins et de 160000 personnes dont seul l'un des deux parents est maghrébin ; 740000 personnes ont les deux parents d'origine européenne et 1,2 millions l'un des deux.

Les travaux portant sur la scolarisation des jeunes issus de l'immigration ont largement commenté l'importance des écarts bruts de performances entre enfants d'immigrés et enfants d'autochtones (Caille, Vallet, 2000 ; Fournier, Silberman, 1999). Ils ont montré que les enfants d'immigrés, qui appartiennent massivement aux milieux sociaux les plus défavorisés et subissent ainsi les contrecoups d'un système scolaire marqué par de fortes inégalités selon l'origine sociale, sont particulièrement vulnérables à l'échec scolaire. Mais ils ont également indiqué que ces différences tendent à disparaître, voire à s'inverser, après contrôle de l'ensemble des variables sociodémographiques. Ainsi, à milieu social identique, les enfants d'immigrés présentent un risque moins élevé de sortie précoce du système éducatif que les élèves de parents français (Caille, 2005). Il n'en reste pas moins qu'ils sont globalement défavorisés pour des raisons qui tiennent à leur origine sociale. Nous souscrivons aux remarques faites par F. Héran pour lequel ce type de méthode et de résultats ne doit pas cacher la réalité sociale vécue par les jeunes issus de l'immigration: "Le fait d'attester que toutes choses égales par ailleurs les enfants d'immigrés font au moins aussi bien que les autres enfants dans les exercices ou les cursus scolaires signifie que les familles immigrées sont à parité avec les autres familles quand on lève l'ensemble des handicaps sociaux qui grèvent leur existence. On apporte ainsi la preuve a contrario que les écarts observés ne sont pas dus à la nature des choses mais à l'inégalité des conditions. [...] Mais cela n'empêche pas que, dans la pratique, l'immigré ou l'enfant d'immigré se caractérise précisément par l'accumulation d'une série de handicaps sociaux qui font partie intégrante de sa condition sociale. » (Héran 2002, p. 32)

Du point de vue du marché du travail, il est aujourd'hui largement démontré que les immigrés, ainsi que les populations issues de l'immigration, sont globalement défavorisés comparativement au reste de la population active (Héran, 2002 ; Dayan, Echardour, Glaude, 1996). Selon l'enquête « Formation et Qualification Professionnelle» de 2003 (FQP 2003, encadré 2), le taux de chômage des immigrés est deux fois plus élevé que celui des Français d'origine (19\% contre $10 \%$ ), alors que celui de leurs descendants directs se situe à un niveau intermédiaire. Les ressortissants du Maghreb sont les plus pénalisés : environ trois actifs sur dix, qu'ils soient immigrés ou issus de l'immigration (ayant leurs deux parents originaires de cette région) sont au chômage. Ceux d'Afrique subsaharienne présentent également des taux de chômage élevés, alors que les immigrés d'origine européenne se situent à un niveau proche de celui 
des Français d'origine. L'effet protecteur du diplôme étant moindre pour les immigrés que pour les Français d'origine, c'est pour les diplômés de l'enseignement supérieur que les différences de taux de chômage sont les plus importantes. Les immigrés ayant acquis la nationalité française sont cependant moins affectés par le chômage (respectivement $16 \%$ contre $21 \%$ pour ceux qui ont conservé leur nationalité d'origine).

Certains travaux ont montré que les écarts entre taux de chômage s'expliquent pour partie par les caractéristiques spécifiques aux immigrés, comme le niveau de diplôme, la qualification et le secteur d'emploi. Selon l'enquête précédente, les immigrés sont plutôt âgés et peu diplômés (personnes sans diplôme ou titulaires au plus d'un brevet des collèges) ; plus du tiers d'entre eux sont ouvriers ou employés non qualifiés. Parallèlement, ceux nés de parents immigrés sont plus jeunes et un peu moins diplômés que les Français d'origine, et leur profession, ainsi que le type d'entreprise dans laquelle ils travaillent, sont relativement proches de ceux des Français d'origine. Néanmoins, un écart important subsiste lorsque l'on neutralise l'impact de ces caractéristiques sur le taux de chômage. De même, comparativement aux Français d'origine, la probabilité de retrouver un emploi est, « toutes choses égales par ailleurs », inférieure pour les étrangers non européens ainsi que pour les Français de parents non européens ${ }^{2}$. En revanche, elle est supérieure pour les étrangers d'origine européenne et les Français d'origine européenne dont la capacité à mobiliser un réseau familial et de connaissances est particulièrement développée (Granovetter 1974; Canamero et alii, 2000).

Les immigrés sont principalement concentrés sur les métiers de premiers niveaux de qualification (ouvriers, employés) les moins susceptibles d'être associés à une formation en cours de carrière. Ils sont également fortement représentés dans les secteurs traditionnellement peu formateurs tels que la construction ou les services aux particuliers. Inversement, ils sont très peu présents dans les secteurs de l'énergie ou des activités financières fortement dispensateurs

\footnotetext{
2 Probabilité de retrouver un emploi 18 mois après une inscription à l'ANPE intervenue au $2^{2}$ trimestre 1995 dans huit zones d'emploi (source : enquête « Trajectoire des demandeurs d'emploi et marché local du travail »). Les variables de contrôle sont le sexe, l'âge, le niveau de formation, la qualification, la durée et le type de contrat du dernier emploi, la région.
}

de formation. Ils occupent également plus souvent des emplois temporaires ou à temps partiel moins concernés par la formation que les emplois stables (Goux, Zamora, 2001).

Si les travaux disponibles permettent de brosser, comme nous venons de le faire très rapidement, un paysage relativement précis de la situation des immigrés sur le marché du travail et, dans une moindre mesure, en entreprise (Bataille, 1997), les études concernant leur accès à la formation continue sont rares, voire inexistantes. Ce manque de données, dans un domaine pourtant par ailleurs largement étudié, peut surprendre. D'autant que depuis la mise en place de la loi fondatrice de juillet 1971 sur la formation professionnelle continue, de nombreuses études ont insisté sur le caractère profondément inégalitaire de l'accès à la formation continue. Ainsi, les travaux issus de l'exploitation des déclarations fiscales des employeurs (encadré 2), des enquêtes auprès des ménages, comme l'enquête «Formation Continue $2000 »$ (FC 2000, encadré 2) ou celles menées auprès des entreprises par Eurostat (Continuing Vocational Training Survey) ont montré que plus le niveau de formation initiale des salariés, la qualification du poste occupé ou la taille des entreprises sont élevés, plus la probabilité d'accès à la formation est importante. Loin de compenser les inégalités enregistrées en formation initiale, le système de formation professionnelle continue a plutôt tendance à les entériner, voire à les accentuer. On retrouve une même tendance à l'inégalité d'accès, bien que de façon atténuée, pour les demandeurs d'emploi (Gelot, Minni, 2004), mais sans que l'on puisse pour autant ici aussi disposer de résultats permettant d'observer d'éventuels écarts entre immigrés et Français d'origine.

Cette inégalité dans l'accès à la formation n'est pas propre à la France (Théry et alii, 2002). Elle a néanmoins conduit les partenaires sociaux à réviser les modalités d'accès des salariés à la formation professionnelle continue ( $c f$. l'Accord national interprofessionnel du 5 décembre 2003, transcrit dans la loi du 4 mai 2004 portant sur « La formation professionnelle tout au long de la vie et le dialogue social »). La création d'un nouveau droit individuel de formation (DIF) a en effet pour objectif de lutter contre les inégalités d'accès à la formation des salariés les moins qualifiés. Les accords de branche, conclus depuis la mise en place de la loi du 4 mai 2004, ont également vocation à cibler les publics prioritaires sur lesquels les bran- 


\section{Encadré 2}

\section{Sources statistiques sur la formation continue et repérage des immigrés}

\section{1 / Les enquêtes Formation Qualification Professionnelle (FQP)}

L'enquête FQP est la principale source disponible en France sur la mobilité sociale et professionnelle. Elle permet, entre autres, de disposer d'informations détaillées sur la formation initiale et continue et la carrière professionnelle des enquêtés. Elle a été conduite à six reprises : en 1964, 1970, 1977, 1985, 1993 et 2003. La dernière enquête a été réalisée entre avril et juillet, auprès de 40000 personnes âgées de 17 à 65 ans, dont 3300 immigrés et 2700 individus issus de l'immigration. L'échantillon de l'enquête réalisée en 1993 ne portait que sur 20000 individus; elle permettait de repérer les seuls immigrés, alors que les enquêtes précédentes ne permettaient aucun repérage de ce type.

Les questions portant en 2003 sur la formation continue concernent les personnes âgées de 17 à 65 ans ayant terminé leurs études initiales. Le repérage des formations porte sur les cinq années qui précédent l'enquête (ou depuis la fin des études initiales si celles-ci se sont terminées il y a moins de 5 ans). On dispose des informations suivantes:

- Ensemble des formations : nombre total de formations suivies ;

- Formations financées par l'employeur (hors alternance) pour les personnes ayant occupé une activité professionnelle (salariés ou indépendants) au cours des 5 dernières années : existence, date, durée, type, spécialité et certification de la formation la plus récente ;

- Formations de plus de 30 heures : existence, date et durée de chacune des formations; financeur, type, spécialité et certification de la formation la plus ancienne;

- Formations en alternance : date, durée, spécialité et certification de la formation la plus ancienne.

Lors de l'enquête réalisée en 1993, ont été recensées les formations suivies depuis la fin des études initiales. On dispose pour cela des informations suivantes:

- Formations financées par l'employeur (hors alternance) : existence, date, durée, intitulé et certification de la formation la plus récente (il est donc possible d'éliminer les personnes dont la dernière formation remonte à plus de 5 ans, et d'être homogène avec l'enquête réalisée en 2003). On dispose également de certaines questions d'opinion sur l'effet de la formation, non disponibles en 2003 ;

- Autres formations (initiative personnelle, CIF (congé individuel de formation), demandeur d'emploi, alternance hors apprentissage) : date, durée, spécialité, certification, et questions d'opinion sur l'effet de la formation.

\section{2 / L'enquête emploi en continue (EEC) et l'enquête sur la Formation continue 2000 (FC 2000)}

Depuis 2002, I'enquête Emploi est réalisée en continue tout au long de l'année auprès d'un échantillon d'environ 300000 personnes, dont 110000 individus différents (l'échantillon de logements est renouvelé par sixième tous les trimestres). On peut repérer les immigrés et, à partir de 2005, ceux issus de l'immigration, mais uniquement pour les personnes en première interrogation. Des questions sont posées sur la formation continue au cours des 3 derniers mois (hors contrats en alternance) : date, durée, type, spécialité et certification.

L'enquête sur la Formation continue, réalisée en mars 2000 (FC 2000), est une enquête complémentaire à l'enquête Emploi qui décrit l'ensemble des formations post-scolaires suivies entre janvier 1999 et février 2000. Elle permet également de repérer les formations suivies de mars à décembre 1998 et de comptabiliser le nombre total de formations dont ont pu bénéficier les personnes interrogées depuis leur sortie de scolarité jusqu'en février 1998. Près de 30000 personnes de moins de 65 ans ont été interrogées. Cette enquête permet de repérer uniquement les immigrés. 
L'écart constaté entre les taux d'accès à la formation en entreprise selon l'enquête FC $2000(26 \%)$ et l'enquête FQP 2003 (34 \%) apparaît faible, compte tenu de la différence de champ temporel de l'observation entre les deux enquêtes: les 11 derniers mois pour l'enquête FC2000, les 5 dernières années pour l'enquête FQP 2003. Cet écart peut s'expliquer en partie par la récurrence des formations. Les personnes qui ont suivi une formation au cours des 5 dernières années en ont pour la plupart suivi d'autres. Mais si l'on se restreint dans FQP aux 11 mois précédant l'enquête (comme dans FC 2000), le taux d'accès est alors de $14 \%$, très inférieur à celui de FC 2000 (26\%). Ce nouvel écart de 12 points pourrait s'expliquer par un effet mémoire : lorsque la dernière formation suivie n'est pas très significative pour la personne interrogée, celle-ci a tendance à décrire une formation plus ancienne et peut donc oublier dans l'enquête FQP certaines formations suivies dans les 11 derniers mois.

\section{3 / Les sources administratives sur la formation continue en entreprise}

Les sources administratives sur la formation continue en entreprise proviennent des déclarations fiscales des employeurs - dites « 24/83», du nom du document utilisé par les entreprises pour leur déclaration annuelle de dépenses de formation, et exploité par le Cereq. Elles ne comportent pas de données fines sur l'origine géographique des salariés concernés.

ches professionnelles devront porter une attention particulière. Mais (faute de données disponibles ?) les immigrés ne constituent pas, en tant que tels, une priorité des partenaires sociaux (Rivier et alii, 2005).

Ce manque d'informations ne peut uniquement s'expliquer par la volonté d'éviter la stigmatisation de certaines populations ou par un souci déontologique en matière de traitements statistiques. On en veut pour preuve qu'en ce qui concerne l'éducation et l'insertion professionnelle des immigrés, les travaux ne manquent pas, même si les données concernant les enfants d'immigrés restent plus rares. L'explication principale réside plutôt dans le fait que nous ne disposions pas, jusqu'à une date récente, de données permettant de combler ce vide ${ }^{3}$. Peu d'enquêtes recensent en effet la nationalité des bénéficiaires de formations, et plus rares encore sont celles permettant de distinguer les immigrés et leurs descendants directs. La dernière enquête FQP, portant sur les formations suivies de 1998 à 2003, est venue combler cette lacune.

Face aux difficultés d'insertion scolaire et professionnelle des immigrés, l'amélioration de leurs compé-

\footnotetext{
${ }^{3}$ Elles impliquent en effet un questionnement sur l'origine des parents, disponible uniquement dans certaines enquêtes spécialisées comme l'enquête «Mobilité géographique et insertion sociale » réalisée en 1992 par l'Insee, l'enquête « Histoire de vie » de 2003, ou à partir d'enquêtes plus générales comme « l'enquête Emploi » (à partir de 2005).
}

tences professionnelles apparaît pourtant essentielle. La formation tout au long de la vie, dont l'un des objectifs affichés est de compenser les inégalités scolaires, devrait jouer un rôle important, en particulier pour les immigrés qui ont rarement accédé à une formation initiale leur permettant de postuler à des emplois qualifiés. Notre propos est donc ici de vérifier si la formation continue joue bien ce rôle de « seconde chance» pour lequel elle est convoquée, ou si elle reproduit les inégalités largement constatées pour d'autres publics en situation défavorable sur le marché du travail. Pour cela, nous procéderons en deux temps.

Dans une première partie, nous nous appuierons sur les taux d'accès des immigrés à la formation continue, qu'il s'agisse des formations dispensées par les employeurs, de celles destinées aux demandeurs d'emploi ou de l'ensemble des actions, quel qu'en soit le mode de financement. Une analyse comparée entre 1993 et 2003 nous permettra de vérifier si l'on peut observer une évolution de cet indicateur sur plus longue période.

Dans un second temps, et afin de tenir compte de la position particulière occupée par les immigrés sur le marché du travail, nous procéderons à une analyse «toutes choses égales par ailleurs». Il s'agira de tenter de dissocier ce qui relève des caractéristiques propres des individus de celles qui renvoient aux politiques de formation (privées ou publiques), voire à la plus ou moins grande « appétence » de certaines catégories de population à se former. 


\section{LES IMMIGRÉS ACCÈDENT PEU À LA FORMATION DANS LE CADRE PROFESSIONNEL}

Les immigrés apparaissent très fortement défavorisés dans l'accès aux formations réalisées dans le cadre professionnel (tableau 1). Les enquêtes disponibles indiquent toutes qu'ils y accèdent environ deux fois moins souvent que les Français d'origine. Ainsi, entre 1998 et 2003, $18 \%$ des immigrés ont suivi au moins une formation, contre $36 \%$ des Français ${ }^{4}$. Si l'on se restreint aux immigrés déjà présents en France en 1998, le taux d'accès reste inchangé, ce qui invalide l'hypothèse selon laquelle l'arrivée de

\footnotetext{
${ }^{4}$ Ont été comptabilisées toutes les personnes ayant occupé un emploi entre 1998 et 2003, quelle que soit leur situation à la date de l'enquête. Ceci explique les taux d'accès différents de ceux présentés dans la publication « Les immigrés en France » (INSEE, 2005) ou seules les personnes occupant un emploi à la date de l'enquête ont été prises en compte.
}

\section{Tableau \\ Taux $d$ 'accès selon les types de formation en $\%$}

\begin{tabular}{|c|c|c|c|c|}
\hline Caractéristiques & $\begin{array}{c}\text { Français } \\
\text { d'origine }\end{array}$ & Immigrés & $\begin{array}{c}\text { Nés de parents } \\
\text { immigrés }\end{array}$ & Ensemble \\
\hline ENSEMBLE DES FORMATIONS & & & & \\
\hline EEC 2004 (3 derniers mois) & 9 & 6 & $\sim$ & 9 \\
\hline FQP 2003 (de 1998 à 2003) & 45 & 27 & 41 & 43 \\
\hline- dont formations supérieures à 30h & 15 & 12 & 16 & 14 \\
\hline FC 2000 (1 1 derniers mois) & 29 & 17 & $\sim$ & 28 \\
\hline FQP 1993 (de 1988 à 1993) & 28 & 16 & $\sim$ & 27 \\
\hline FORMATION EN ENTREPRISE & & & & 8 \\
\hline EEC 2004 (3 derniers mois) & 8 & 4 & 31 & 34 \\
\hline FQP 2003 (de 1998 à 2003) & 36 & 18 & 30 & 32 \\
\hline- dont salariés du privé & 48 & 17 & 43 & 47 \\
\hline- dont salariés du public & 19 & 9 & 18 & 18 \\
\hline- dont indépendants & 27 & 15 & $\sim \sim$ & 26 \\
\hline FC 2000 (1 1 derniers mois) & 24 & 8 & $\sim \sim$ & 22 \\
\hline FQP 1993 (de 1988 à 1993) & & & & \\
\hline FORMATION DES CHÔMEURS & 4 & 4 & $\sim$ & 13 \\
\hline EEC 2004 (3 derniers mois) & 13 & 10 & $\sim$ & \\
\hline FC 2000 (1 1 derniers mois) & & & $\sim$ & \\
\hline
\end{tabular}

: non disponible jusqu'en 2005, année à partir de laquelle l'enquête Emploi renseigne sur le pays et la nationalité à la naissance des parents de la personne interrogée.

$\sim$ : non disponible

Champ commun : individus ayant terminé leur formation initiale, âgés de 17 à 64 ans.

Champ des formations EEC : ENTREPRISE, personnes en emploi à la date de l'enquête, formations proposées par l'employeur.

CHOMEURS, personnes au chômage à la date de l'enquête, formations proposées par l'ANPE ou un autre organisme d'insertion.

Champ des formations en ENTREPRISE FQP : formations financées ou proposées par l'employeur pour les personnes ayant eu au moins un emploi au cours des 5 années d'observation de l'enquête. Le statut (salarié du privé, du public ou indépendant) correspond au statut à la date de l'enquête ou lors du dernier emploi occupé.

Champ des formations FC 2000 : les formations sont définies par rapport à la situation de l'individu le mois précédent l'entrée en formation (en emploi ou chômeur), appréhendée par le calendrier de l'enquête Emploi.

Exemple de lecture : selon l'enquête FQP 2003, $17 \%$ des immigrés salariés du privé ont suivi au moins une formation financée ou proposée par leur employeur au cours des 5 dernières années, contre $35 \%$ pour les Français d'origine, $30 \%$ pour ceux nés de parents immigrés et $32 \%$ en moyenne.

Source : FQP 1993 et 2003, EEC 2004, FC 2000 (exploitation auteurs). 
certains d'entre eux après cette date pourrait expliquer le moindre accès de l'ensemble des immigrés à la formation. Qu'ils soient salariés du public, du privé ou travailleurs indépendants, les immigrés restent défavorisés, même si l'écart est un peu moindre pour les salariés de l'État ou des collectivités territoriales ${ }^{5}$.

En revanche, il n'en est pas de même pour les formations qui s'adressent aux chômeurs, difficilement appréhendables à partir de l'enquête FQP, pour lesquelles les enquêtes EEC 2004 et FC 2000 ne montrent pas de différences significatives selon l'origine ethnique des bénéficiaires.

Si l'on se limite aux formations de plus de 30 heures, particulièrement intéressantes du point de vue de notre problématique étant donné leur caractère qualifiant et/ou professionnalisant, la différence est de moindre ampleur. Deux éléments contribuent à expliquer ce faible écart. D'une part, les immigrés sont plus souvent au chômage, et l'on sait que les formations suivies par les demandeurs d'emploi sont en moyenne quatre fois plus longues que les formations en entreprise (Gelot, Minni, 2004). Par ailleurs, les immigrés au chômage suivent des formations plus longues que les Français d'origine. Ainsi, selon l'enquête FC 2000, huit formations sur dix suivies par des immigrés à la recherche d'un emploi durent plus de 30 heures, contre six sur dix pour les Français d'origine. Ceci laisse à penser que les populations les plus démunies en capital scolaire, professionnel ou culturel, bénéficient d'une attention particulière de la part des services en charge de la politique de l'emploi. En revanche, les formations en entreprise ont une durée proche, quelle que soit l'origine du bénéficiaire.

Sur les dix dernières années, conformément aux évolutions de l'économie et à la demande des entreprises, le taux global d'accès à la formation, ainsi que celui des formations financées par les employeurs, tendent à progresser fortement, aussi bien pour les Français d'origine que pour les immigrés ; cela révèle une prise en compte plus forte de la problématique de la formation dans le déroulement des carrières. Les écarts entre ces deux populations restent assez stables sur l'ensemble de la période, même si l'on peut cons-

\footnotetext{
${ }^{5}$ Il s'agit là d'immigrés ayant acquis la nationalité française.
}

tater un léger resserrement pour les formations financées par les employeurs ${ }^{6}$.

Comparativement à leurs ascendants, la situation des enfants d'immigrés apparaît beaucoup moins contrastée. Selon l'enquête FQP, leur probabilité d'accéder à une formation est toujours assez proche de celle des Français d'origine. Ces résultats sont confirmés par une première exploitation de l'EEC 2005 sur un échantillon restreint d'individus ${ }^{7}$, qui ne montre pas de différence significative entre descendants directs d'immigrés et Français d'origine.

\section{DES INÉGALITÉS D'ACCÉS À LA FORMATION COMME POUR LE RESTE DE LA POPULATION}

Les inégalités d'accès à la formation, déjà largement constatées pour l'ensemble des salariés, se vérifient également pour les immigrés (tableau 2). Les différenciations selon le sexe, l'âge ou le niveau de diplôme sont reproduites, quelle que soit l'origine ethnique. Ainsi, les hommes, les jeunes et les plus diplômés accèdent plus souvent aux actions de formation que l'ensemble de la population, qu'elle soit immigrée ou Française d'origine. Il n'en est pas de même pour les chômeurs pour lesquels ces critères apparaissent peu discriminants.

Les écarts constatés entre immigrés et Français d'origine sont également confirmés quels que soient l'âge, le sexe ou le diplôme: les immigrés enregistrent toujours des taux d'accès à la formation bien inférieurs à ceux des Français d'origine, alors que ceux issus de l'immigration se situent à un niveau moyen.

Nous avons déjà rappelé que l'investissement des entreprises en matière de formation varie fortement selon les secteurs professionnels auxquels elles sont rattachées. Traditionnellement, les entreprises fortement utilisatrices de main-d'œuvre non qualifiée

\footnotetext{
${ }^{6}$ La probabilité pour un immigré de suivre une formation en entreprise, versus de ne pas en suivre, est comparativement à un français d'origine de 0,29 en 1993 (odds ratio). Elle progresse à 0,38 en 2003. Pour l'ensemble des formations, elle est respectivement de 0,49 et 0,45 .

7 Sixième entrant des deux premiers trimestres 2005, pour lequel on connaît le pays d'origine et la nationalité de naissance des parents.
} 
Tableau 2

Taux d'accès selon le sexe, l'âge, le diplôme et le type de formation en $\%$

\begin{tabular}{|c|c|c|c|c|}
\hline Caractéristiques & Français d'origine & Immigrés & Nés de parents immigrés & Ensemble \\
\hline \multicolumn{5}{|c|}{ ENSEMBLE DES FORMATIONS - FQP 2003} \\
\hline \multicolumn{5}{|c|}{ SEXE } \\
\hline Hommes & 48 & 29 & 44 & 46 \\
\hline Femmes & 42 & 25 & 40 & 41 \\
\hline \multicolumn{5}{|l|}{$\hat{A} G E$} \\
\hline 17-29 ans & 53 & 38 & 45 & 51 \\
\hline $30-49$ ans & 51 & 32 & 48 & 49 \\
\hline $50-65$ ans & 30 & 16 & 26 & 28 \\
\hline \multicolumn{5}{|l|}{ DIPLÔME } \\
\hline Supérieur & 63 & 45 & 62 & 61 \\
\hline Secondaire (bac et CAP-BEP*) & 48 & 38 & 55 & 47 \\
\hline Peu diplômé (brevet et non diplômé) & 32 & 18 & 30 & 29 \\
\hline Total & 45 & 27 & 41 & 43 \\
\hline \multicolumn{5}{|c|}{ FORMATIONS EN ENTREPRISES - FQP 2003} \\
\hline \multicolumn{5}{|c|}{ SEXE } \\
\hline Hommes & 38 & 18 & 33 & 35 \\
\hline Femmes & 35 & 18 & 29 & 33 \\
\hline \multicolumn{5}{|l|}{$\hat{A} G E$} \\
\hline $17-29$ ans & 35 & 20 & 26 & 33 \\
\hline $30-49$ ans & 41 & 20 & 36 & 39 \\
\hline $50-65$ ans & 28 & 13 & 26 & 27 \\
\hline \multicolumn{5}{|l|}{ DIPLÔME } \\
\hline Supérieur & 50 & 32 & 48 & 49 \\
\hline Secondaire (bac et CAP-BEP) & 37 & 21 & 31 & 36 \\
\hline Peu diplômé (Brevet et non diplômé) & 26 & 12 & 22 & 24 \\
\hline 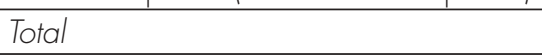 & 36 & 18 & 31 & 34 \\
\hline \multicolumn{5}{|c|}{ FORMATIONS DES CHÔMEURS - EEC 2004} \\
\hline \multicolumn{5}{|c|}{ SEXE } \\
\hline Hommes & 4 & 4 & $\sim$ & 4 \\
\hline Femmes & 4 & 4 & $\sim$ & 4 \\
\hline \multicolumn{5}{|l|}{$\hat{\mathrm{A} G E}$} \\
\hline $17-29$ ans & 4 & 5 & $\sim$ & 4 \\
\hline $30-49$ ans & 5 & 4 & $\sim$ & 4 \\
\hline $50-65$ ans & 4 & 2 & $\sim$ & 3 \\
\hline \multicolumn{5}{|l|}{ DIPLÔME } \\
\hline Supérieur & 4 & 3 & $\sim$ & 4 \\
\hline Secondaire (bac et CAP-BEP) & 4 & 5 & $\sim$ & 4 \\
\hline Peu diplômé (Brevet et non diplômé) & 3 & 3 & $\sim$ & 3 \\
\hline Total & 4 & 4 & $\sim$ & 4 \\
\hline
\end{tabular}

* : respectivement certificat d'aptitude professionnelle et brevet d'études professionnelles.

: non disponible jusqu'en 2005, année à partir de laquelle l'enquête Emploi renseigne sur le pays et la nationalité à la naissance des parents de la personne interrogée.

Champ commun : individus âgés de 17 à 64 ans ayant terminé leur formation initiale.

Source : FQP 2003 (exploitation auteurs). 
dans des secteurs comme la construction ou les services affichent un effort de formation particulièrement faible. Dès lors, il apparaît utile de mesurer de manière plus approfondie leur comportement, sachant qu'elles emploient une part importante de salariés immigrés ou issus de l'immigration. L'exploitation de l'enquête FQP 2003 montre que, quelle que soit la profession, la taille ou le secteur de l'établissement employeur, la participation des immigrés à la formation en entreprise reste très inférieure à celle enregistrée pour les Français nés de parents français (tableau 3). Dans certains secteurs, tel que la construction par exemple, elle peut être quatre fois plus faible pour les immigrés que pour les Français d'origine. Dans ces secteurs, peu formateurs, la situation des immigrés dans leur ensemble apparaît donc particulièrement défavorable. Leur très faible taux d'accès à la formation accentue les difficultés traditionnelle- ment rencontrées par ces populations sur le marché $\mathrm{du}$ travail et rend beaucoup plus difficile toute perspective de mobilité professionnelle ascendante.

\section{UN ACCÉS RELATIVEMENT UNIFORME SELON L'ORIGINE GÉOGRAPHIQUE}

Au-delà d'une analyse concernant l'ensemble des immigrés, quel que soit le pays de naissance, les enquêtes disponibles ne montrent pas de différenciation importante selon l'origine géographique. En effet, qu'ils soient originaires du Maghreb, du reste de l'Afrique, d'Europe ou de certains pays du SudEst asiatique, les immigrés accèdent de manière relativement uniforme aux différentes actions de formation continue, en particulier pour la formation

Tableau 3

Taux d'accès à la formation en entreprise selon les caractéristiques du dernier emploi en $\%$

\begin{tabular}{|c|c|c|c|c|}
\hline Caractéristiques & $\begin{array}{l}\text { Français } \\
\text { d'origine }\end{array}$ & Immigrés & $\begin{array}{l}\text { Nés de parents } \\
\text { immigrés }\end{array}$ & Ensemble \\
\hline \multicolumn{5}{|l|}{ PROFESSION } \\
\hline Indépendants & 18 & 8 & 19 & 17 \\
\hline Professions supérieures & 51 & 38 & 51 & 51 \\
\hline Professions intermédiaires & 49 & 34 & 43 & 48 \\
\hline Employés qualifiés & 41 & 26 & 35 & 40 \\
\hline Employés non qualifiés & 21 & 12 & 19 & 20 \\
\hline Ouvriers qualifiés & 30 & 13 & 25 & 28 \\
\hline Ouvriers non qualifiés & 14 & 9 & 11 & 13 \\
\hline \multicolumn{5}{|l|}{ SECTEUR D'ACTIVITÉ } \\
\hline Agriculture & 15 & 14 & 10 & 15 \\
\hline Industrie & 36 & 20 & 32 & 34 \\
\hline Construction & 24 & 7 & 21 & 21 \\
\hline Tertiaire & 39 & 20 & 33 & 37 \\
\hline \multicolumn{5}{|l|}{ TAILLE D'ÉTABLISSEMENT } \\
\hline Moins de 10 salariés & 18 & 8 & 13 & 17 \\
\hline 10 à 49 salariés & 27 & 11 & 19 & 25 \\
\hline 50 à 199 salariés & 35 & 17 & 33 & 33 \\
\hline 200 salariés et + & 48 & 30 & 43 & 46 \\
\hline Ensemble & 36 & 18 & 31 & 34 \\
\hline
\end{tabular}

Champ : individus âgés de 17 à 64 ans ayant terminé leur formation initiale et ayant occupé au moins un emploi au cours des 5 années d'observation de l'enquête.

Source : FQP 2003, (exploitation auteurs). 
réalisée dans le cadre professionnel. Selon l'enquête FQP 2003, les taux d'accès à la formation en entreprise passent de $17 \%$ pour les Maghrébins à $21 \%$ pour les Asiatiques, contre $36 \%$ pour les Français d'origine. Ces écarts relativement faibles sont confirmés par les résultats de l'enquête EEC 2004 (tableau 4).

Ainsi, l'ensemble des personnes nées de parents immigrés accèdent aux actions de formation continue à un niveau proche de celui des Français d'origine ; il n'en va pas de même pour ceux dont les deux parents sont originaires d'Afrique du Nord. Ils accèdent en effet rarement à la formation dans le cadre de l'entreprise : seuls $23 \%$ d'entre eux ont suivi une formation entre 1998 et 2003, contre $31 \%$ pour l'ensemble des enfants d'immigrés. Un tel résultat va dans le sens des travaux portant sur l'accès à la formation en alternance qui montrent un moindre accès des jeunes maghrébins à l'apprentissage (Fournier, Silberman, 1999). Cette situation est d'autant plus problématique que les jeunes d'origine maghrébine, et particulièrement les jeunes hommes, sont souvent en situation d'échec scolaire (Lainé, Okba, 2005 ; Caille, 2005). On pouvait attendre de la formation continue qu'elle joue ce rôle de " seconde chance " pour lequel elle est convoquée et compense ainsi une absence de capital social et culturel particulièrement préjudiciable à ce type de population ; cela n'apparaît pas être le cas. En revanche, les personnes dont seul l'un des parents est originaire du Maghreb accèdent autant à la formation en entreprise que les Français d'origine (tableau 4). Un tel phénomène a également été constaté par d'autres auteurs concernant le risque relatif d'être au chômage selon le pays d'origine des parents (Meurs, Pailhé, Simon, 2005). Ils ont montré que les enfants des couples mixtes sont en situation intermédiaire par

Tableau 4

Taux d'accès à la formation selon l'origine géographique

en $\%$

\begin{tabular}{|l|c|c|}
\hline Caractéristiques & $\begin{array}{c}\text { Ensemble des } \\
\text { formations }\end{array}$ & $\begin{array}{c}\text { Formations en } \\
\text { entreprise }\end{array}$ \\
\hline IMMIGRÉS FQP 2003 & 27 & 18 \\
\hline Ensemble des immigrés & 24 & 17 \\
\hline dont Maghreb & 34 & 19 \\
\hline dont Afrique subsaharienne & 24 & 11 \\
\hline dont Sud-Est asiatique & 26 & \\
\hline dont Europe & & 4 \\
\hline IMMIGRÉS EEC 2004 & 6 & 4 \\
\hline Ensemble des immigrés & 6 & 3 \\
\hline dont Maghreb & 7 & 6 \\
\hline dont Afrique subsaharienne & 6 & 5 \\
\hline dont Sud-Est asiatique & 6 & 31 \\
\hline dont Europe & & 23 \\
\hline NÉS DE PARENTS IMMIGRÉS FQP 2003 & 41 & 37 \\
\hline Ensemble des descendants d'immigrés & 42 & 32 \\
\hline dont 2 parents maghrébins & 42 & 33 \\
\hline dont 1 parent maghrébin, 1 parent français & 42 & \\
\hline dont 2 parents d'origine européenne & 41 & \\
\hline dont 1 parent d'origine européenne, 1 parent français & & \\
\hline
\end{tabular}

Origines géographiques : Maghreb (Algérie, Maroc, Tunisie); Afrique subsaharienne (autres pays d'Afrique) ; Sud-Est asiatique (Cambodge, Laos, Vietnam); Europe (Continent européen). Sont présentes dans ce tableau uniquement les origines géographiques pour lesquelles on dispose dans l'enquête FQP 2003 d'un nombre suffisant d'observations pour assurer la représentativité des résultats.

Source : FQP 2003, EEC 2004 (exploitation auteurs). 
rapport aux Français d'origine et à ceux dont les deux parents sont immigrés.

\section{DES FORMATIONS MOINS NOMBREUSES MAIS PLUS QUALIFIANTES}

Les écarts constatés entre les immigrés, leurs descendants directs et les Français d'origine concernant le taux d'accès à la formation de l'ensemble des 17-65 ans, qu'ils soient en emploi, au chômage, ou inactifs, se vérifient également lorsque l'on s'intéresse au nombre de formations suivies. Ainsi, parmi ceux qui ont suivi au moins une action de formation entre 1998 et 2003, et quel qu'en soit le type, sept immigrés sur dix n'ont bénéficié que d'une ou deux formations, contre environ six Français d'origine sur dix (tableau 5). La situation des descendants d'immigrés reste, comme précédemment, plus proche de celle des Français d'origine que de celle des immigrés euxmêmes. Ils sont presque aussi nombreux que les Français d'origine à avoir suivi plus de deux formations dans les cinq années précédant l'enquête.

L'observation du nombre de formations suivies est utile à la compréhension du phénomène étudié ; cependant, elle demeure incomplète et mérite d'être appréciée également du point de vue de la durée des formations dispensées. Pour ceux qui bénéficient d'une action de formation, la durée de celle-ci permet en effet de mieux qualifier l'investissement réalisé par les personnes et les employeurs. Pour ce faire, l'enquête FQP 2003 indique la durée de la dernière formation suivie en entreprise et celle de toutes les formations de longue durée (plus de 30 heures).
Selon ces deux critères, qui renvoient en quelque sorte à la qualité de la formation dispensée, la relation est inversée. Ce sont alors les immigrés qui apparaissent les plus favorisés. En effet, la durée moyenne de la dernière formation suivie en entreprise est deux fois plus longue pour les immigrés (200 heures) que pour leurs descendants directs ou pour les Français d'origine. Quant à l'ensemble des formations de longue durée, quel qu'en soit leur mode de financement (essentiellement public), leur durée cumulée dépasse 600 heures en moyenne pour les immigrés, contre environ 450 heures pour celles dispensées aux enfants d'immigrés ainsi qu'aux Français d'origine. Un tel processus a d'ailleurs été observé pour les chômeurs qui, bien qu'accèdant mois souvent que les salariés à la formation, cumulent des durées de stage nettement plus longues (Gelot, Minni, 2004). Ce constat en appelle un autre: les formations suivies dans le cadre du plan de formation en entreprise par les immigrés sont également plus certifiantes ${ }^{8}$ : c'est le cas de $16 \%$ d'entre elles, contre $9 \%$ des formations suivies par les Français d'origine ou les personnes issues de l'immigration. De ce point de vue, tout fonctionne comme si l'effort des employeurs, comme celui des services en charge de la réinsertion des demandeurs d'emploi, se centrait sur un nombre réduit d'individus mais que les personnes concernées bénéficiaient en revanche d'une attention soutenue de la part de ces deux principaux financeurs de la formation continue. Ce résultat semble apporter un regard complémentaire (et plutôt inverse) à celui

\footnotetext{
${ }^{8}$ Sont considérées ici comme certifiantes les formations qui permettent l'obtention d'un diplôme ou d'un titre homologué.
}

Tableau 5

Nombre total de formations (1998-2003)

en $\%$

\begin{tabular}{|l|c|c|c|c|}
\hline & 1 ou 2 formations & 3 formations & 4 formations et plus & Total \\
\hline Français d'origine & 57 & 25 & 17 & 100 \\
\hline Immigrés & 70 & 18 & 12 & 100 \\
\hline Nés de parents immigrés & 61 & 23 & 16 & 100 \\
\hline Ensemble & 59 & 25 & 17 & 100 \\
\hline
\end{tabular}

Champ : personnes ayant suivi au moins une formation au cours des 5 dernieres années, quel qu'en soit le type.

Source : FQP 2003, (exploitation auteurs) 
porté par les études menées en France sur les discriminations à l'embauche, et en particulier sur le rôle des intermédiaires entre demandeurs et offreurs de travail, qui tendent à conclure à un rôle discriminatoire conscient ou inconscient de leur part en matière d'embauche (Deroche, Viprey, 1997 ; Noël, 2000).

\section{UN MOINDRE ACCÉS À LA FORMATION QUI PERSISTE}

La statistique descriptive nous a montré que la situation des immigrés vis-à-vis de la formation professionnelle ne suit pas une logique d'ensemble. Si les immigrés sont désavantagés du point de vue de l'accès à la formation professionnelle continue, il n'en est pas de même pour les personnes nées de parents immigrés, pour lesquelles on observe des taux d'accès proches de ceux des Français nés de parents français, à l'exception de ceux dont les deux parents sont maghrébins.

Néanmoins, comme l'a montré la littérature (Héran, 2002), les immigrés occupent une place spécifique sur le marché du travail. Le fait qu'ils disposent d'un niveau de formation inférieur à la moyenne, qu'ils soient concentrés dans certains secteurs, qu'ils occupent les emplois les plus précaires et les moins qualifiés nécessite de s'interroger sur le lien de causalité entre caractéristiques individuelles et accès à la formation en cours de carrière professionnelle. Autrement dit, leur faible accès aux dispositifs de formation post-scolaire s'explique-t-il par leurs caractéristiques individuelles et/ou par leur positionnement sur le marché du travail ? Ou bien existe-t-il une réelle pénalité d'accès à la formation en fonction de l'origine ethnique ou géographique?

Pour tenir compte de manière synthétique de l'ensemble de ces effets de structure, nous avons procédé à une analyse «toutes choses égales par ailleurs». Pour les différentes catégories de population étudiées, nous avons estimé par un modèle « logit » la probabilité relative de bénéficier d'une action de formation professionnelle continue en entreprise, en contrôlant un certain nombre de variables (observables) sociodémographiques et d'autres relatives aux caractéristiques des entreprises.

Le résultat de cette approche économétrique confirme les résultats observés précédemment. On constate ainsi (tableau 6) que la sous-exposition des immigrés au bénéfice de la formation, observée dans les données brutes, est conservée après contrôle des effets de structure. Lorsque l'on prend en compte les caractéristiques individuelles, ainsi que celles du dernier emploi et de l'entreprise où il est exercé, la probabilité d'accès à la formation professionnelle reste plus faible pour les immigrés, quelle que soit leur origine et, à un niveau moindre, pour les descendants des immigrés dont les deux parents sont maghrébins. Ce résultat est confirmé lorsque l'on distingue parmi les non-diplômés ou titulaires d'un CEP (nombreux parmi les immigrés), ceux qui n'ont pas été scolarisés, de ceux qui n'ont fréquenté que l'école primaire, le collège sans avoir dépassé la classe de 4 è, ou enfin ceux qui ont eu une scolarité plus longue. Par ailleurs, si l'on se restreint aux seuls salariés de l'État ou des collectivités locales, la probabilité d'accès à la formation reste moindre pour les immigrés, mais la pénalité ethnique des jeunes issus de l'immigration maghrébine disparaît.

$\mathrm{Au}$ vu de ces différents résultats, une première conclusion s'impose : alors que pour l'accès à l'emploi les phénomènes de discrimination touchent en premier lieu les immigrés originaires du Maghreb, pour la formation professionnelle, c'est bien l'ensemble des immigrés qui apparaissent défavorisés, quelle que soit leur origine, même si ce phénomène persiste pour les seuls descendants d'immigrés dont les parents sont originaires du Maghreb.

Parmi les différentes variables qui n'ont pu être prises en compte dans le modèle, celle liée à l'attente des salariés vis-à-vis de la formation joue un rôle important. Autrement dit, nous devons tenter de répondre à la question suivante : le moindre accès des immigrés à la formation peut-il s'expliquer (au moins en partie) par leur moindre appétence (Fournier, 2004) ou doiton le rechercher dans les pratiques développées par certains employeurs? De ce point de vue, l'enquête FC 2000 apporte de précieuses informations. Elle indique que les immigrés occupant des postes non qualifiés déclarent des besoins de formation non satisfaits un peu supérieurs à ceux des Français d'origine de même qualification, alors que leur taux d'accès est très inférieur (tableau 7). Elle montre également qu'ils sont proportionnellement légèrement moins nombreux à refuser une formation. Cela invite plutôt à trancher dans le sens d'une plus grande appétence des immigrés pour la formation car en général des besoins de formation non satisfaits 
Tableau 6

\section{Les facteurs de l'accès à la formation professionnelle}

\begin{tabular}{|c|c|c|c|}
\hline & \begin{tabular}{|c|} 
Écart par rapport \\
à la probabilité de \\
référence $(40 \%)$
\end{tabular} & & $\begin{array}{l}\text { Écart par rapport } \\
\text { à la probabilité de } \\
\text { référence }(40 \%)\end{array}$ \\
\hline Origine ethnique & & Employés non qualifiés du privé & -9 \\
\hline Français d'origine & Référence & Ouvriers qualifiés du public & -8 \\
\hline Immigrés : Europe & -11 & Ouvriers qualifiés du privé & -12 \\
\hline Immigrés: Amérique & -17 & Ouvriers non qualifiés du public & -16 \\
\hline Immigrés: Maghreb & -11 & Ouvriers non qualifiés du privé & -22 \\
\hline Immigrés: Afrique subsaharienne & -11 & Durée d'emploi de 1998 à 2003 & \\
\hline Immigrés : Asie du Sud-Est & -9 & 60 mois & Référence \\
\hline Immigrés: Autres & -13 & de 49 à 59 mois & -6 \\
\hline Nés de parents immigrés : 2 parents & -8 & de 25 à 48 mois & -15 \\
\hline maghrébins & -8 & moins de 25 mois & -24 \\
\hline $\begin{array}{l}\text { Nés de parents immigrés: } 1 \text { parent } \\
\text { maghrébin, } 1 \text { parent trançais d'origine }\end{array}$ & 0 & Travail à temps partiel & \\
\hline Nés de parents immigrés : 2 parents & & Temps complet & Référence \\
\hline $\begin{array}{l}\text { européens } \\
\text { ens }\end{array}$ & -4 & \begin{tabular}{|l|} 
Temps partiel \\
\end{tabular} & -4 \\
\hline Nés de parents immigrés: 1 parent & & Type de contrat & \\
\hline européen, 1 parent français d'origine & -3 & À durée indéterminée & Référence \\
\hline Nés de parents immigrés: autres & 0 & Temporaire (intérim et CDD) & -8 \\
\hline Sexe & & Secteur d'activité & \\
\hline Hommes & Référence & Agriculture, sylviculture & $\sim$ \\
\hline Femmes & -2 & IAA (industries agroalimentaires) & $\sim$ \\
\hline Diplôme & & Biens de consommation & Référence \\
\hline Supérieur & 6 & Automobile & 10 \\
\hline Baccalauréat & 4 & Biens d'équipement & 10 \\
\hline CAP, BEP & Référence & Biens intermédiaires & 4 \\
\hline Brevet & $\sim$ & Énergie & 17 \\
\hline CEP, sans diplôme & -8 & Construction & $\sim$ \\
\hline Âge & & Commerce et réparation & $\sim$ \\
\hline Jeunes (moins de 30 ans) & 5 & Transports & 13 \\
\hline Adultes (30 à 49 ans) & Référence & Activités financières & 15 \\
\hline Seniors (50 ans et plus) & -11 & Activités immobilières & 8 \\
\hline Profession & & Services aux entreprises & 7 \\
\hline Indépendants et professions libérales & -16 & Services aux particuliers & $\sim$ \\
\hline Professions supérieures du public & -5 & Éducation, santé, action sociale & 11 \\
\hline Professions supérieures du privé & $\sim$ & Administrations & 11 \\
\hline Professions intermédiaires du public & $\sim$ & Taille de l'établissement & \\
\hline Professions intermédiaires du privé & Référence & moins de 10 salariés & -8 \\
\hline Employés qualifiés du public & $\sim$ & 10 à 49 salariés & Référence \\
\hline Employés qualifiés du privé & -7 & 50 à 199 salariés & 8 \\
\hline Employés non qualifiés du public & -10 & 200 salariés et plus & 16 \\
\hline
\end{tabular}

$\sim$ : non significatif.

Champ : individus ayant terminé leur formation initiale, âgés de 17 à 64 ans, et ayant occupé au moins un emploi au cours des 5 années d'observation de l'enquête.

Exemple de lecture : un homme immigré d'origine maghrébine, diplômé d'un CAP/ BEP, âgé de 30 à 49 ans, ayant travaillé de façon continue de 1998 à 2003 et occupant à la date de l'enquête un emploi intermédiaire à temps plein et à durée indéterminée dans un établissement de 10 à 49 salariés du secteur des biens de consommation, a une probabilité d'avoir suivi au moins une formation professionnelle de $29 \%$ contre $40 \%$ pour un Français d'origine présentant les mêmes caractéristiques. L'écart des probabilités (-11\%) est significatif au seuil de $5 \%$.

Source : enquête FQP 2003, (exploitation auteurs). 


\section{Tableau 7 \\ Besoins, refus et attentes de formation en entreprise \\ en $\%$}

\begin{tabular}{|l|c|c|c|c|}
\hline & Ensemble & $\begin{array}{c}\text { Professions } \\
\text { supérieures et } \\
\text { intermédiaires }\end{array}$ & $\begin{array}{c}\text { Employés } \\
\text { et ouvriers } \\
\text { qualifiés }\end{array}$ & $\begin{array}{c}\text { Employés } \\
\text { et ouvriers } \\
\text { non qualifiés }\end{array}$ \\
\hline Besoins de formation non satisfaits & & & & \\
\hline Français d'origine & 25 & 31 & 22 & 18 \\
\hline Immigrés & 23 & 31 & 19 & 23 \\
\hline Formation refusée par l'individu & & & & \\
\hline Français d'origine & 5 & 6 & 5 & 4 \\
\hline Immigrés & 3 & 7 & 3 & 3 \\
\hline Besoins importants de formation dans le futur & & & & \\
\hline Français d'origine & 61 & 71 & 59 & 48 \\
\hline Immigrés & 44 & 66 & 40 & 36 \\
\hline
\end{tabular}

Champ : individus âgés de 17 à 64 ans ayant terminé leur formation initiale, et salariés à la date de l'enquête.

Note de lecture : $22 \%$ des employés et ouvriers non qualifiés immigrés déclarent des besoins de formation non satisfaits entre janvier 1999 et février 2000, $3 \%$ ont refusé une proposition de formation et $36 \%$ expriment des besoins importants dans le futur.

Source : enquête FC2000, (exploitation auteurs).

importants vont de pair avec un taux d'accès élevé ( $c f$. Fournier C., article à paraître en 2006 dans Formation Emploi). Ce résultat éclairerait alors d'un jour nouveau l'argument de la distance culturelle, maintes fois invoqué dans la littérature pour expliquer les discriminations existantes en matière d'emploi à l'égard des populations immigrées.

Cette première approche de l'appétence semble néanmoins relativisée par l'étude des besoins futurs exprimés par cette catégorie de population. Les immigrés déclarent en effet moins souvent ressentir de besoins importants de formation dans le futur que les Français d'origine. C'est surtout le cas pour ceux qui ont déclaré ne pas avoir de besoins insatisfaits. Ainsi, parmi les immigrés non qualifiés qui sont dans ce cas, $23 \%$ expriment des besoins futurs importants, contre $41 \%$ des Français d'origine de même niveau professionnel. Cette apparente contradiction peut s'expliquer par un effet de structure. En effet, les attentes en termes de formation décroissant avec l'âge ( $75 \%$ des moins de 30 ans déclarent des besoins importants dans le futur, contre 30\% des 50-64 ans) et les immigrés étant plus âgés que les Français d'origine, un tel comportement est de ce point de vue compréhensible. Un écart important entre immigrés et Français d'origine persiste néanmoins après contrôle de cette variable.
Mais plus fondamentalement, cette apparente contradiction entre besoins non satisfaits et attentes futures renvoie selon nous à la difficulté à cerner la notion « d'appétence » qui demande, au-delà des approches par questionnaires, que soient développées des études de nature sociologique plus qualitatives, propices à l'analyse d'une notion polysémique. L'appétence en matière de formation continue, comme dans d'autres domaines, est avant tout un construit social qui renvoie à la vision que peuvent avoir différentes catégories de population des possibilités qui leur sont ouvertes, ainsi qu'au contexte professionnel dans lequel elles évoluent. Ces possibilités sont ellesmêmes le fruit de leur propre histoire scolaire, professionnelle et sociale. Pour reprendre ce que Demazière et Dubar nomment «l'univers des croyances » ou « le monde des possibles » lorsqu'ils analysent les déterminants des parcours des jeunes non qualifiés, on peut y voir la conséquence de leurs expériences antérieures dans le domaine de la formation continue (Demazière, Dubar, 1997).

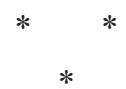

À ce stade de l'analyse, nos résultats peuvent être éclairés par les travaux étrangers sur cette question controversée. Ainsi, Lochhead (2002) montre qu'au 
Canada les salariés d'immigration récente enregistrent un taux d'accès à la formation financée par les employeurs nettement plus faible que celui des immigrés les plus anciens ou des personnes nées au Canada. Tout comme dans le cas français, ce résultat se vérifie, quelles que soient les caractéristiques des salariés, des entreprises ou des types de contrat de travail. Une régression logistique multivariée, contrôlant ce type de variables, confirme que le statut d'immigré récent est toujours associé à un faible taux d'accès à la formation financée par les employeurs. Mais pour l'auteur, ce moindre accès ne peut s'expliquer par une moindre appétence de ces salariés pour la formation. Au contraire, les sources disponibles tendent à montrer, comme c'est le cas pour la France, que les salariés d'immigration récente sont moins enclins à refuser une formation qui leur est proposée que les natifs du pays ${ }^{9}$. Ils sont également relativement plus nombreux que la population de référence à engager des formations en lien avec l'emploi occupé, même si elles ne sont pas financées par leur employeur.

L'exploitation de l'enquête FQP et de l'enquête Emploi a constitué une occasion nouvelle de disposer d'informations détaillées sur l'accès des immigrés à la formation et de compléter ainsi la connaissance de ce domaine. Nous avons pu vérifier que, là comme ailleurs, des inégalités perdurent, alors que la loi de 1971 avait pour objectif de les réduire. Une analyse économétrique du taux de participation à la formation nous a permis de confirmer ce résultat. Une fois

${ }^{9}$ Il s'agit ici de l'ensemble des formations, quel que soit le financeur. contrôlées les caractéristiques des individus et celles des entreprises dans lesquelles ils travaillent, les immigrés, ainsi que les enfants d'immigrés dont les deux parents sont maghrébins, connaissent toujours une moindre probabilité d'accès à la formation professionnelle. Nous avons néanmoins montré que les immigrés qui ont pu accéder à une formation en cours d'emploi, ou durant une période de chômage, bénéficient d'actions plus qualifiantes que leurs homologues Français d'origine. C'est sans doute dans un tel résultat que nous pouvons trouver des éléments d'explication du faible taux d'accès des immigrés à la formation: celui-ci se situerait à l'intersection de deux logiques en partie divergentes, celle des entreprises qui privilégient les formations courtes d'adaptation au poste de travail et celle des immigrés dont les besoins se portent sur des formations plus longues de remise à niveau ou de qualification préalables à l'accès à des formations professionalisantes.

La loi du 4 mai 2004 sur la formation professionnelle tout au long de la vie ne pouvant cibler explicitement ces catégories de population, il y a fort à craindre que cette situation ne connaisse pas d'amélioration rapide. Si, comme nous l'avons indiqué plus haut, les besoins de formation dépendent du contexte professionnel et de la connaissance que les salariés peuvent avoir des possibilités existantes de se former, il n'en demeure pas moins qu'une politique ciblée sur les publics exclus de la formation, notamment les moins qualifiés, qui allierait obligation de financer et obligation de former (droit inversement proportionnel au niveau de formation), permettrait de réduire en partie les écarts d'accès à la formation professionnelle entre immigrés et non-immigrés.

\section{Bibliographie}

Bataille P. (1997), Le racisme au travail, Éditions La Découverte.

Caille J.-P., Vallet L.-A. (2000), «La scolarité des enfants d'immigrés", in Van Zanten A. (dir.), L'école: l'état des savoirs, Paris, La Découverte, pp. 293-301.
Caille J.-P. (2005), « Scolarisation et diplômes obtenus en fin de scolarité de jeunes issus de l'immigration », Actes du Colloque L'insertion professionnelle des jeunes issus de l'immigration, Dares - Ined, Paris.

Canamero C., Canceil G., Cloarec N. (2000), «Chômeurs étrangers et chômeurs d'origine 
étrangère ", Premières synthèses, Dares, $\mathrm{n}^{\circ}$ 46-2, novembre.

Chazal J. (2005), Les demandeurs d'emploi étrangers, collection « Les essentiels », Observatoire de l'ANPE.

Dayan J.-L., Echardour A., Glaude M. (1996), «Le parcours professionnel des immigrés en France : une analyse longitudinale», Économie et Statistique, $\mathrm{n}^{\circ} 299$, Insee, Paris.

Demazière D., Dubar C. (1997), Analyser les entretiens biographiques, l'exemple de récits d'insertion, Nathan.

Deroche L., Viprey M. (1997), «Recrutement et discriminant des firmes à l'égard de la main-d'œuvre étrangère ou issue de l'immigration ", in Le racisme et les discriminations au travail, Colloque IMA, $\mathrm{n}^{\circ} 158 / \mathrm{a}$.

Fournier I., Silberman R. (1999), « Les enfants d'immigrés sur le marché du travail: les mécanismes d'une discrimination sélective », Formation Emploi $\mathrm{n}^{\circ} 65$, janvier-mars, pp. 31-55.

Fournier C. (2004), « Aux origines de l'inégale appétence des salariés pour la formation» Bref-Cereq $\mathrm{n}^{\circ} 209$, juin.

Gelot D., Minni C. (2004), « Les chômeurs accèdent deux fois moins que les salariés à la formation continue, mais pour des durées plus longues", Premières synthèses, Dares $n^{\circ}$ 51.2, décembre.

Goux D., Zamora P. (2001), « La formation en entreprise continue de se développer », Insee Première $\mathrm{n}^{\circ} 759$, février.

Grannovetter M-S. (1974), Getting a job. A study of Contacts and carreers, Harvad University Press, Cambridge.
Héran F. (2002), Immigration, marché du travail, intégration, Rapport du séminaire du Commissariat Général au Plan, La Documentation française.

INSEE (2005), Les immigrés en France, Collection « Références ».

Lainé F., Okba M. (2005), « Les jeunes issus de l'immigration : de l'école au métier », Travail et Emploi, $\mathrm{n}^{\circ} 103$, Dares.

Lochhead C. (2002), Employment sponsored training among recent immigrants, Canadian Labour and Business Centre.

Meurs D., Pailhé A., Simon P. (2005), Immigrés et enfants d'immigrés sur le marché du travail: une affaire de génération?, Colloque Ined/Dares.

Ministère de l'Aménagement du territoire, de la Ville et de l'Intégration, Direction de la population et des migrations (1997), «L'insertion professionnelle des étrangers : emploi, chômage, évolution de 1992 à 1996 et dispositifs d'insertion en 1995 », Notes et documents, $\mathrm{n}^{\circ} 33$, avril.

Noël O. (2000), « La face cachée de l'intégration : les discriminations institutionnelles à l'embauche », VEI Enjeux, ${ }^{\circ} 121$, juin.

Rivier C., Seiler C. (2005), L'évaluation de la mise en oeuvre des accords de branche sur la formation professionnelle tout au long de la vie résultant de l'ANI du 20 septembre 2003 transposé dans la loi du 4 mai 2004, Rapport Eurostat / Dares sous la direction de J.-M. Luttringer.

Théry M., Rousset P., Zygmunt C. (2002), « L'Europe de la formation tout au long de la vie reste à construire », Cereq-Bref, n 187.

Tribalat M. (1989), « Immigrés, étrangers, français : l'imbroglio statistique », Population et Sociétés $n^{\circ} 241$, INED. 


\title{
Résumé
}

\section{Les immigrés accèdent moins à la formation professionnelle continue}

\author{
Didier Gélot et Claude Minni
}

L'article s'intéresse à l'accès des immigrés à la formation professionnelle continue. II s'appuie sur des données issues de plusieurs enquêtes, dont notamment l'enquête "Formation et qualification professionnelle (FQP) » qui permet, pour la première fois en 2003, de repérer, outre les immigrés, leurs descendants directs.

Comme l'ensemble de la population, les immigrés et leurs descendants directs les plus âgés et les moins qualifiés connaissent des difficultés d'accès à la formation. L'article pointe par ailleurs un moindre accès à la formation professionnelle continue de l'ensemble des immigrés (quelle que soit leur origine géographique) et de leurs descendants lorsque leurs parents sont originaires du Maghreb.

\section{Mots clés}

Travailleur migrant, FPC - formation professionnelle continue, accès à la FPC, enquête FQP.

Journal of Economic Literature : J 15, J 71. 